Mots. Les langages du politique

$88 \mid 2008$

Discours politique, discours expert

\title{
La dépolitisation du discours au sein des rapports annuels de l'Organisation mondiale du commerce
}

Jean-Louis Siroux

\section{OpenEdition}

Journals

Édition électronique

URL : https://journals.openedition.org/mots/14223

DOI : $10.4000 /$ mots. 14223

ISSN : 1960-6001

Éditeur

ENS Éditions

Édition imprimée

Date de publication : 1 novembre 2008

Pagination : 13-23

ISBN : 978-2-84788-147-9

ISSN : 0243-6450

Référence électronique

Jean-Louis Siroux, "La dépolitisation du discours au sein des rapports annuels de l'Organisation mondiale du commerce », Mots. Les langages du politique [En ligne], 88 | 2008, mis en ligne le 01 novembre 2010, consulté le 23 avril 2022. URL : http://journals.openedition.org/mots/14223 ; DOI https://doi.org/10.4000/mots.14223 


\section{La dépolitisation du discours au sein des rapports annuels de l'Organisation mondiale du commerce}

Chaque année, l'Organisation mondiale du commerce (OMC) publie un rapport annuel afin de dresser le bilan de son activité et de faire le point sur la situation du commerce mondial. En marge des textes juridiques, souvent volumineux et ésotériques (comme les accords commerciaux et leur réglementation), le rapport annuel a pour objectif de rendre compte de l'action de l'OMC à un public plus large que les seuls membres des gouvernements nationaux. Ces rapports, rédigés par le secrétariat de l'OMC et qui s'adressent aussi bien, par exemple, à des journalistes qu'à des ONG, comprennent généralement 150 à 200 pages et représentent la vitrine extérieure de l'organisation.

Dans le cadre de cet article, nous nous intéresserons plus spécifiquement aux quelques pages qui introduisent chaque rapport. Dans un court chapitre de 5 ou 6 pages, intitulé "Tour d'horizon», l'OMC livre un bref aperçu de ses analyses et de ses activités. Ce «Tour d'horizon » ne constitue pas pour autant, comme son intitulé pourrait le laisser supposer, une synthèse sommaire ou un simple bilan factuel de l'année écoulée. Le texte est, au contraire, doté d'une forte dimension normative dans la mesure où il s'agit d'un texte politique, issu d'une organisation politique et destiné à être suivi d'effets politiques. Toutefois, comme bon nombre de publications de grandes organisations internationales, le discours de l'OMC ${ }^{1}$ cherche à se présenter sous un jour impartial. Les arguments paraissent relever de l'évidence et sont dès lors présumés objets de consensus. Doté d'une forte autorité symbolique, le locuteur parait s'élever doctement au-dessus des querelles partisanes qui agitent les acteurs de la vie politique.

Nous montrerons dans cet article que les rapports annuels de l'OMC appartiennent à cette catégorie de discours judicieusement décrite par Pierre Bourdieu comme des «discours politiques dépolitisés» (Bourdieu, 1982, p. 155). Bien que ses prises de position et ses recommandations soient éminemment politiques, l'OMC les présente soit comme relevant du bon sens, soit

Université catholique de Louvain, GRAID, siroux@anso.ucl.ac.be

1. Les rapports annuels de l'OMC sont ici désignées sous le sigle «RA» (suivi de l'année et de la page). 
comme la conséquence logique d'une expertise éclairée. Elles apparaissent dès lors non pas comme une simple option politique, mais comme une évidence ou une nécessité. C'est en référence à cette neutralisation de la dimension politique du discours que sera mobilisé ici le concept de « discours politique dépolitisé».

À partir d'un corpus formé par les «Tours d'horizon» de 1998 à $2004^{2}$, je procéderai à une analyse critique de ce discours en vue de repérer quelques procédés discursifs par lesquels l'OMC parvient à défendre un véritable programme politique sans jamais le présenter comme tel3.

\section{La naturalisation des enjeux politiques dans le discours}

La dissimulation des enjeux politiques du discours s'exprime sous une première forme, celle du recours à diverses figures de style comme la métaphore et l'analogie.

La mobilisation de métaphores peut, il est vrai, se révéler d'une réelle utilité heuristique. Elles permettent de mettre en exergue des caractéristiques ou des mécanismes qui n'apparaissent guère à travers la désignation commune d'un phénomène. De fait, l'usage de certains types de métaphores constitue un précieux indicateur de l'orientation générale d'un discours. Tel nous parait être le cas des trois principaux types repérés au sein de ce corpus.

On relève tout d'abord un nombre considérable de verbes de mouvement et de termes évoquant une évolution linéaire et mécanique. Ralentissement, reprise, rythme, stagnation, accélération ou passage à la vitesse supérieure constituent autant de formes nominales ou syntagmes suggérant l'évolution saccadée mais continue vers un objectif déjà fixé. Qu'elle freine ou qu'elle s'accélère, l'histoire que nous décrit l'OMC garde toujours en point de mire un horizon déterminé.

Le deuxième type de métaphores, sanitaire, concourt également à légitimer, au moyen de l'expertise médicale, les objectifs économiques prônés par l'organisation. L'économie connait parfois quelques défaillances qu'il convient de traiter avec la médication adéquate.

Il est essentiel de continuer résolument à libéraliser le commerce pour que l'économie mondiale se rétablisse [...] Le commerce mondial des marchandises et des services est l'appareil circulatoire de l'économie mondiale. Si l'appareil est défaillant, la santé du patient ne peut pas s'améliorer, quel que soit le médicament macro-économique prescrit. (RA 1998, 2)

2. Soit un corpus de 3832 formes et de 27282 occurrences.

3. L'analyse qui suit a été facilitée par l'utilisation du logiciel de lexicométrie Lexico 3, réalisé par l'équipe du SYLED de l'Université Sorbonne nouvelle Paris 3. 
Enfin, l'OMC a également recours à un troisième type de métaphores d'ordre naturaliste. Les crises économiques, les récessions ou les politiques générales forment autant de phénomènes naturels qui agissent, interagissent et astreignent les hommes à s'adapter. Une crise économique devient ainsi « une tourmente financière » qui «s'est abattue sur une bonne partie du monde », une récession «apparait à l'horizon » et la mondialisation constitue un «processus entrainé par un courant de changement technologique et économique».

Le terme mondialisation désigne toute une série de tendances observées dans les domaines de l'économie, de la technologie et des relations internationales, qui peuvent se renforcer mutuellement mais qui ont des origines diverses [...] Le monde a toujours connu des périodes d'effondrement rapide, des obstacles à l'activité économique et au contact humain. (RA 2000,4)

Au travers de ce discours, la mondialisation constitue une réalité floue, insaisissable, mais inhérente au destin de l'humanité. Il ne s'agit donc pas, comme le précise explicitement l'OMC, d'un programme - qui serait soumis à l'approbation des électeurs - mais d'un processus inscrit dans le devenir de l'humanité, dont les origines sont incertaines. Toutefois, « devant la confusion et les craintes que [la mondialisation] suscite abondamment dans le public, les gouvernements et les institutions internationales doivent élaborer un programme positif s'ils veulent éviter une réaction populiste et protectionniste» (RA 2000, 5). La nuance est de taille. La mondialisation n'est pas un programme volontariste, mais elle requiert un programme pour faire face à ses conséquences.

\section{L’expert et le politique}

Ces «processus», ces «tendances» ou ces «tourmentes financières» constituent quelques-uns des sujets des rapports annuels de l'OMC. Le discours se veut universel, global; il émane de tous, c'est-à-dire de personne en particulier. Les rares acteurs auxquels il est fait allusion sont, dans l'ordre de fréquence décroissante, les «membres de l'OMC» (sans distinction), les «ministres», le «directeur général», les «gouvernements», le «président du conseil», le "secrétariat», les «organisations internationales», les «comités», les «groupes» («spéciaux», «parlementaires», « de travail»), les "parlementaires», les «participants», les «délégations». Sont ainsi identifiés des groupes d'individus, de pays ou d'organisations évoqués sans distinction, dotés d'intérêts que l'on suppose communs ou distingués par des désaccords ne pouvant être que provisoires.

La dénégation du pluralisme politique trouve en réalité ici l'une de ses expressions les plus importantes. Les actions menées par l'organisation, nous l'avons déjà souligné, paraissent susciter un consensus qu'aucune tension 
sociale ni aucun conflit politique ne parviennent à troubler4. Or, en présentant ses objectifs comme figés et consensuels, en les présentant comme inéluctables, l'OMC ne se contente pas de défendre un programme politique particulier, mais esquisse peu à peu une véritable redéfinition de ce qu'est un pouvoir politique. La question des moyens se substitue à celles des fins et la question du «comment» à celle du «quoi » (et du «pourquoi»)5.

L'OMC peut aider à apporter une réponse au problème central de la gestion publique de notre nouvelle ère mondiale: le fait que les gouvernements répondent principalement à leurs mandants, alors que de plus en plus le système économique doit répondre aux besoins mondiaux. (RA 1998, 4)

Lorsque la légitimité du représentant démocratique disparait au profit de l'efficacité de l'expert, les gouvernements se transforment en acteurs de second rôle chargés de faire preuve de bonne volonté collective, sinon en gestionnaires qui s'appliquent, de façon plus ou moins heureuse, à adapter les législations nationales aux besoins de l'économie mondiale. La sagesse des gouvernements et des parlements nationaux semble en effet se mesurer à l'aune de leur capacité de résistance au protectionnisme et de leur rapidité d'ajustement aux règles de l'OMC.

[...] On ne peut que conclure que, face à cette crise, les gouvernements se sont comportés de manière raisonnable [...]. (RA 1999, 2)

Dans plusieurs cas, cela n'a pas encore eu lieu (en raison principalement de lenteurs ou d'une inaction sur le plan législatif lorsque la mesure en question et incompatible avec les règles de l'OMC est une disposition légale). (RA 2004, 5)

\section{Des chiffres qui ne trompent pas}

Cette technicisation du discours repose essentiellement sur la mobilisation d'indicateurs quantitatifs chargés d'orienter et de cautionner les orientations politiques.

L'OMC fait référence à de nombreuses reprises aux données statistiques émises par la Banque mondiale (BM), l’Organisation de coopération et de développement économiques (OCDE) ou le Fonds monétaire international (FMI), avalisant par là, au moyen d'un jeu de balancier, les objectifs, communs pour la plupart, de ces organisations. Un large chapitre intitulé tantôt «perspective économique », tantôt « perspective du commerce»-les deux formules

4. En témoigne par exemple la mobilisation d'expressions telles que volonté collective, volonté commune, volonté générale.

5. L'OMC n'a évidemment pas le monopole de cette redéfinition technocratique du pouvoir politique. On retrouve ainsi, de manière relativement récurrente, au sein des documents émis par l'Union européenne, un processus de dénégation du conflit au profit d'une rhétorique du consensus et du dialogue (voir Gobin, 2004 et Cussó, Dufresne et al., 2008). 
étant souvent utilisées comme synonymes, de sorte que l'économie parait se limiter à la seule mesure des échanges commerciaux -, fait ainsi état des prédictions du FMI ou de la BM pour l'année à venir. Guidés par les chiffres de la croissance 6 , les «prévisionnistes», comme les nomme joliment le rapport de 1999, soulignent les bienfaits occasionnés par les mesures de libéralisation et l'intérêt à poursuivre en ce sens.

Lorsqu'elles ne proviennent pas directement d'autres organisations internationales, les études empiriques auxquelles fait référence l'OMC sont des études sans signataires. L'OMC renvoie à «certaines estimations», à «la théorie», à «ces études» ou à «de multiples études». Elle se réfère par là à ce qui a déjà été démontré, à ce qui a déjà été démenti, bref à ce qui ne doit plus l'être. La puissance exemplative du chiffre se substitue à des démonstrations plus exigeantes, davantage sujettes à la réfutation ou à la controverse politique.

On comprend mieux, dès lors, que la polémique suscitée par les critiques des mouvements altermondialistes fasse moins l'objet d'une véritable récusation que d'une fin de non-recevoirt. En réalité, ces critiques relèvent aux yeux de l'OMC de deux ordres distincts. Les premières sont dues à la mauvaise foi des contestataires qui contraste avec la «volonté collective des membres d'aller de l'avant de façon constructive» et avec l'intérêt manifesté par "le public ». Elles ne sont, dès lors, nullement présentées comme une option politique qui mériterait d'être débattue - et éventuellement combattue -, mais comme une analyse incorrecte ne pouvant susciter qu'un démenti.

Il est légitime que des opinions divergent sur ce que devraient être les politiques des pays dans ces secteurs sensibles, mais il est vrai aussi qu'on a relevé divers commentaires infondés et une certaine désinformation cependant que le public prenait de plus en plus conscience de l'importance de ces négociations. (RA 2001, 2)

Les secondes relèvent quant à elles davantage de la méprise et sont présentées comme étant à la source d'une interprétation précipitée et d'une confusion quant au véritable rôle joué par l'OMC.

Ce qui s'est passé à Seattle a été largement commenté dans les médias et dans le feu de l'action il y a eu quelques déclarations catégoriques sur l'utilité et l'avenir de l’OMC. Les interprétations les plus alarmistes des évènements de Seattle ont déjà été démenties. (RA 2000, 2)

Le dogmatisme (des déclarations "catégoriques») et l'emportement de l'adversaire («dans le feu de l'action») mettent d'entrée ses idées hors jeu et dédouanent le locuteur de toute contre-argumentation. À la confrontation

6. Le terme croissance apparait 62 fois dans le corpus et est utilisé à 15 reprises en l'espace de 37 lignes au sein du rapport de 1999.

7. Notons que tel est également le cas des critiques des «pays en développement » dont les réticences sont attribuées à un déficit d'intégration et de compréhension. 
démocratique des idées se substitue la récusation des idées de l'adversaire, jugées non redevables d'une véritable réfutation.

\section{Les mots du discours}

La naturalisation du discours, nous l'avons souligné, a pour effet de présenter une réalité déproblématisée et dépourvue de toute ambigüité. Les évènements se succèdent «naturellement», selon une logique jamais prise en défaut. Roland Barthes le soulignait déjà dans Mythologies:

En passant de l'histoire à la nature, le mythe fait une économie: il abolit la complexité des actes humains, leur donne la simplicité des essences, il supprime toute dialectique, toute remontée au-delà du visible immédiat, il organise un monde sans contradiction parce que sans profondeur, un monde étalé dans l'évidence, il fonde une clarté heureuse. Les choses ont l'air de signifier toutes seules. (Barthes, 1957, p. 231)

La dimension conflictuelle et la part de contingence propre à toute science (et donc aux sciences économiques...) tend ainsi à disparaitre lorsque le discours extrait le propos de la sphère du discutable. Le recours à un champ lexical spécifique et positivement connoté, bien que très imprécis, aboutit à un résultat relativement analogue.

La répétition d'un vocabulaire difficilement assimilable à une réalité empirique particulière encourage, en effet, une lecture passive et inattentive. Les affirmations s'enchainent et se renforcent mutuellement de sorte que l'on finit par ne plus très bien savoir si telle critique "déjà démentie» l'a effectivement été. Le sentiment de cohérence que procure la construction circulaire du discours (la croissance engendre le développement qui est source de croissance...) suscite l'adhésion à un discours qui semble tourner autour de luimême. Cet effet de fermeture se caractérise notamment par l'appréciation ou la dépréciation de certains termes au moyen d'un environnement lexical particulièrement limité.

Le terme mesure, par exemple, est utilisé dans le jargon de l'OMC en référence à toute décision ou à tout acte législatif, quel que soit le niveau de pouvoir dont il émane. Les «mesures» renvoient autant aux engagements pris par l'OMC dans le but d' «aider les pays en développement» qu'aux législations nationales qui représentent un obstacle à la libéralisation du commerce. Le caractère vague et générique de la notion conduit à distinguer les réalités très différentes auxquelles elle renvoie au moyen de leur environnement lexical. Il y a les bonnes et les mauvaises mesures, aisément différenciables d'après la nature de leurs conséquences.

Aucun des pays affectés par la crise financière n'a eu recours au protectionnisme et bon nombre d'entre eux ont en fait pris des mesures audacieuses pour conti- 
nuer d'ouvrir leur marché. En outre, leurs partenaires commerciaux se sont également montrés déterminés à résister aux pressions protectionnistes. Les quelques mesures commerciales qui ont été prises par un petit nombre de pays n'ont pas suffi à enrayer la tendance à la poursuite de la libéralisation découlant de la mise en œuvre des résultats du cycle de l'Uruguay, et dans certains cas, des initiatives autonomes des gouvernements. ${ }^{8}$ (RA 1999, 2)

Dans cet extrait, la première occurrence renvoie aux «mesures» édictées en vue d'ouvrir les marchés. Elles sont «audacieuses» et se situent aux antipodes d'éventuelles mesures protectionnistes. La seconde se rapporte par contre à «quelques mesures» marginales («prises par un petit nombre de pays») édictées en vue de nuire («enrayer») mais inefficaces face à la volonté collective de libéraliser (elles «n'ont pas suffi »).

Entièrement construits autour d'une opposition binaire (la libéralisation d'un côté, le protectionnisme de l'autre), les rapports de l'OMC présentent une réalité limpide car dépourvue de toute ambigüité. Des «pressions protectionnistes » suscitent des «difficultés », une libéralisation des politiques commerciales engendre une "avancée». Les premières produisent des crises, les secondes y remédient. Le monde devient aisément intelligible.

Conformément aux principes fondateurs de l'OMC, la libéralisation apparait en outre comme une évolution nécessaire, inéluctable même, mais toujours incomplète. Il s'agit bien d'une action en train de se faire dont on connait l'importance (elle est "ambitieuse », « indispensable »), mais à laquelle on ne fixe guère de limite. L'économie n'est jamais libéralisée, elle est toujours en cours de libéralisation. La libéralisation est présentée comme un processus au long cours, au sein duquel chaque nouveau round de négociations ne constitue qu'une petite pierre posée sur l'édifice. La libéralisation n'est jamais gagnée, on la «poursuit», on la « réactive», on la «maintient», on la «consolide », on l' "accroit» ou on en "accélère le rythme». Chaque mesure de libéralisation est un «effort». Quand des «efforts» ont été accomplis, il s'agit d'un "progrès», d'une "amélioration». Plusieurs "progrès» engendrent quant à eux un «succès ». Et un «succès» en appelle d'autres, dans d'autres domaines. Chaque année semble dès lors constituer une époque charnière :

Pour l'OMC, l'année 2001 a surtout été l'année de la Conférence ministérielle de Doha, qui a marqué un tournant dans l'histoire de l'OMC et dans les relations entre les pays développés et les pays en développement. (RA 2002, 2)

L'année 2002 a été une période d'intense activité pour l'Organisation mondiale du commerce. (RA 2003, 2)

Révélateur de l'accent mis sur le caractère linéaire et évolutif de l'histoire, l'adverbe plus apparait pas moins de 252 fois dans notre corpus, souvent

\section{C'est moi qui souligne.}


précédé des adverbes bien ou beaucoup; tout y est «beaucoup plus important», "beaucoup plus vaste », «beaucoup plus urgent», «beaucoup plus fortement», "bien plus élevés», "bien plus libérales», etc. La locution de plus en plus apparait quant à elle 18 fois, soulignant le chemin parcouru vers une «économie mondiale de plus en plus interdépendante» ou vers «un monde de plus en plus intégré».

D'autre part, si l'OMC use abondamment, dans l'ensemble de ses rapports, du terme libéralisation (29 occurrences), il est intéressant de remarquer que la référence au libéralisme comme doctrine est, en revanche, absente. La libéralisation parait ne découler d'aucune théorie politique. Et si, comme le souligne le rapport de 2000, «la mondialisation n'est pas un programme », la libéralisation ne parait pas l'être davantage.

Le terme protectionnisme fait par contre bien partie du lexique de l'OMC. Cité à cinq reprises, il est présenté comme une doctrine à laquelle on «a recours », à laquelle on « résiste» ou que l'on « rejette». Plus significatif, l'adjectif protectionniste est utilisé quatre fois sur six au sein du syntagme les pressions protectionnistes. Alors que la libéralisation s'impose par la force de l'évidence, le protectionnisme doit sa puissance de conviction à des pressions dont on connait la cible mais dont on ignore l'origine. Tel est par exemple le cas de ces pressions qui «continueront d'être exercées sur les gouvernements pour qu'ils prennent des mesures protectionnistes». De qui ces pressions émanent-elles? Des syndicats? Des partis politiques? Une fois de plus, aucun acteur n'est identifié.

Précisons enfin que si les pressions protectionnistes s'abattent sur les gouvernements, tels la grippe ou le mauvais temps, la libéralisation s'accomplit elle aussi sans le concours de l'homme. Si libéralisation est souvent jumelé à des verbes sanctionnant l'évolution (s'accroitre, se poursuivre...), le verbe libéraliser, conjugué ou non, n'apparait quant à lui qu'à une seule reprise. Le monde se libéralise tout seul.

\section{Discours autorisé et autorité du discours}

En définitive, le discours de l'OMC revêt un apparent paradoxe. Il apparait, en effet, au terme de cette brève analyse, qu'il entretient de nombreuses similitudes avec le discours d'autres organisations internationales. L'impersonnalité des constructions verbales et nominales, l'absence d'acteurs identifiés, une apparente neutralité, la propension à naturaliser les rapports sociaux et le recours à un champ lexical vague et positivement connoté paraissent caractéristiques de cette langue mondiale qu'ont disséquée de multiples travaux. Il suffit de se référer à l'ouvrage coordonné par Gilbert Rist pour constater les similitudes qui unissent le discours de l'OMC à celui de la BM ou de l'Organi- 
sation des nations unies (Rist, 2002). La prétendue impartialité qu'est censée assurer un discours suffisamment vague et détaché de référence sociohistorique pour se prémunir de toute réfutation représente une sorte de fil rouge qui traverse les rapports des organisations internationales.

Toutefois, nous avons également souligné le caractère éminemment normatif d'un discours politiquement et idéologiquement situé dans la mesure où l'Organisation mondiale du commerce a pour fonction même de promouvoir la libéralisation des échanges commerciaux. Cependant, les «Tours d'horizon » opérés par le secrétariat survolent un espace si étroit et s'opèrent avec une telle absence de nuances et de modération que l'on en vient à s'interroger sur cet étonnant paradoxe: comment un discours doté d'une telle dimension militante parvient-il à se présenter sous les atours de l'analyse impartiale et non partisane, redevable à la seule évidence de ses observations? Ou plutôt, d'où tient-il l'autorité nécessaire pour reposer sur une contradiction aussi manifeste tout en prétendant demeurer crédible?

Cette question mériterait sans doute des développements théoriques qui déborderaient le cadre de cet article. Il est toutefois intéressant, afin de suggérer quelques pistes de réflexion, d'opérer un très bref détour par les notions de capacité technique et de capacité statutaire développées par Pierre Bourdieu (1982).

Deux types d'interprétation sont en effet généralement avancés lorsque l'on tente d'identifier l'origine de l'autorité d'un discours. Une première interprétation (Austin, 1991) cherche le pouvoir des mots dans la forme même du discours. Certains énoncés, par leur qualité stylistique, par leur puissance suggestive ou incitative, seraient dotés d'une autorité intrinsèque. La capacité technique du locuteur, c'est-à-dire son habilité à s'exprimer selon les normes en vigueur, à prendre en compte la dimension rituelle du discours, à s'exprimer avec aisance et charisme, serait déterminante dans le processus de légitimation du discours.

Une seconde interprétation privilégie quant à elle les conditions de production du discours. La compétence technique du locuteur dépendrait certes de son habilité linguistique, mais surtout de son habilitation à en faire usage, c'est-à-dire de sa capacité statutaire. Ainsi, d'après Bourdieu, «l'autorité advient au langage du dehors »:

Cette autorité, le langage tout au plus la représente, il la manifeste, il la symbolise: il y a une rhétorique caractéristique de tous les discours d'institution, c'est-à-dire de la parole officielle du porte-parole autorisé s'exprimant en situation solennelle, avec une autorité qui a les mêmes limites que la délégation de l'institution.9 (Bourdieu, 1982, p. 105-107)

9. Il serait intéressant de compléter cette analyse par une étude plus spécifique des conditions de production (et d'écriture en particulier) des rapports annuels de l'OMC, de manière assez similaire à l'analyse proposée par Alexandre Duchêne du discours onusien (Duchêne, 2004). 
Ces deux grilles de lecture ne constituent pas deux modèles dichotomiques et inconciliables. On peut au contraire défendre l'idée que ces deux formes d'autorité, technique et statutaire, constituent deux dimensions complémentaires du processus de légitimation du discours: le locuteur transfère son autorité au discours qui, par sa conformité aux lois du genre, atteste à son tour de l'autorité du locuteur.

S'il est en effet peu probable qu'un discours tel que celui de l'OMC puisse conserver une quelconque légitimité sous la plume d'un locuteur non autorisé, il n'est toutefois pas davantage évident que celle-ci jouisse de l'autorité nécessaire pour dire n'importe quoi, n’importe comment. Chaque genre a ses lois, ses rituels, qui le rendent prévisibles et qui permettent de le distinguer des autres genres. Méconnaitre ces lois ou les ignorer, c'est forcément mettre à nu une part de son autorité, l'exposer et, en définitive, la mettre en jeu.

C'est ainsi que le discours expert, que beaucoup d'auteurs comparent avec raison à la novlangue de Georges Orwell, peut être considéré comme une variété particulière, dont les caractéristiques se reproduisent d'un discours à l'autre, d'une organisation à l'autre. En réalité, comme l'observe Gilbert Rist, le seul fait de publier des discours tenus pour légitimes, sous une forme qui l'est également, atteste de l'autorité du locuteur (Rist, 2002, p. 18). De quel crédit bénéficierait, en effet, une organisation internationale reconnue si elle se mettait à enfreindre ouvertement les lois du genre, à dire ce qui ne se dit pas et à le dire de la mauvaise manière? La légitimité statutaire enjoint le recours à des formes d'expression pourvues d'une certaine légitimité. Si la compétence statutaire est à l'origine de la compétence linguistique, elle s'accompagne toutefois le plus souvent de la capacité technique, «ne serait-ce que parce qu'elle en commande l'acquisition par l'effet de l'assignation statutaire (noblesse oblige)»(Bourdieu, 1982, p. 64).

Cependant, il parait tout aussi improbable qu'un discours, conforme en tout point aux normes en vigueur, puisse conserver quelque crédit sous la plume d'un locuteur non autorisé. Prononcé par un locuteur ordinaire, il serait probablement dépourvu de toute légitimité, tant l'écart statutaire qui le séparerait des conditions d'expression légitimes serait important. Un discours bâti, comme nous l'avons vu, autour de l'argument d'autorité n'a en effet de sens que s'il est prononcé par quelqu'un jouissant de l'autorité nécessaire pour émettre un tel propos sans jamais avoir à le justifier (est-il fondé, pertinent?) ni à se justifier (suis-je autorisé à le prononcer?). Il ne sera donc doté d'une certaine autorité que si le locuteur est autorisé à le prononcer, et à le prononcer de cette manière. Dans le cas contraire, l'effet réel risque de ne pas correspondre à l'effet voulu.

En définitive, il est possible de voir dans ce rapport de réciprocité, entre capacités technique et statutaire, l'un des fondements de l'autorité du dis- 
cours de l'OMC. Le discours tient certes une part de son autorité à sa conformité aux lois du genre, mais ces lois, faites de reconnaissance tacite davantage que d'imposition expresse, ne peuvent être consacrées que de façon circulaire, par ceux qui détiennent l'autorité suffisante pour le faire. Or, à force de mobiliser le même lexique, les mêmes arguments ou les mêmes statistiques, au prix de paraphrases et d'incessants renvois d'ascenseur, les grandes organisations internationales en arrivent à présenter comme évident ce qui ne relève pourtant que d'une option politique, nécessairement sujette à caution. Et à présenter comme inéluctable ce qui, par définition, ne l'est pas.

\section{Références}

AUSTIN John Langshaw, 1991, Quand dire, c'est faire, Paris, Le Seuil.

BARThes Roland, 1957, Mythologies, Paris, Le Seuil.

Bourdieu Pierre, 1982, Ce que parlerveut dire, Paris, Fayard.

Cussó Roser, DufreSne Anne et al., 2008, Le conflit social éludé, Louvain-la-Neuve, Academia-Bruylant.

DuCHÊNE Alexandre, 2004, "Construction institutionnelle des discours. Idéologies et pratiques dans une organisation supranationale», Travaux neuchâtelois de linguistique, $\mathrm{n}^{\circ}$ 40, p. 93-115.

Gobin Corinne, 2004, "Gouverner par les mots. Des stratégies lexicales au service du consensus... contre le social?», Éducation et sociétés, n¹3, p. 85-101.

RIST Gilbert éd., 2002, Les mots du pouvoir. Sens et non-sens de la rhétorique internationale, Genève, IUED (Enjeux). 